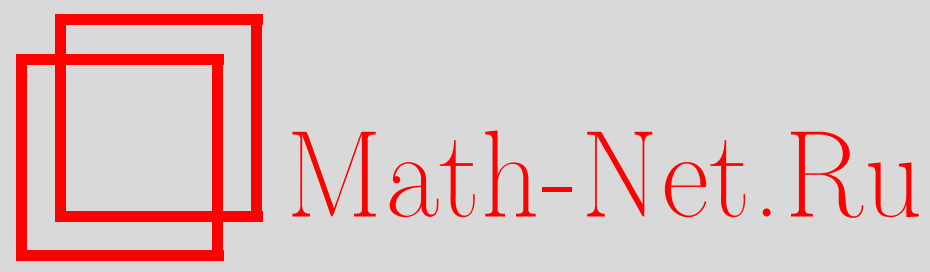

У. Г. Абдуллаев, О мгновенном сжатии носителя решения нелинейного вырождающегося параболического уравнения, Матем. заметки, 1998, том 63, выпуск 3, 323-331

DOI: https://doi.org/10.4213/mzm1286

Использование Общероссийского математического портала Math-Net.Ru подразумевает, что вы прочитали и согласны с пользовательским соглашением http://www.mathnet.ru/rus/agreement

Параметры загрузки:

IP : 54.92 .164 .108

26 апреля 2023 г., 16:16:19

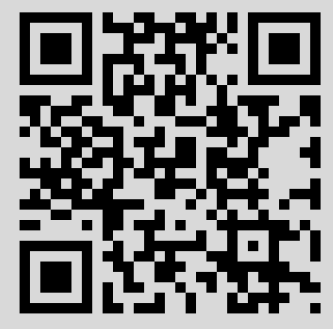




\section{О МГНОВЕННОМ СЖАТИИ НОСИТЕЛЯ РЕШЕНИЯ НЕЛИНЕЙНОГО ВЫРОЖДАЮЩЕГОСЯ ПАРАБОЛИЧЕСКОГО УРАВНЕНИЯ}

\section{У. Г. Абдуллаев}

Изучается эффект сжатия носителя решения нелинейного параболического уравнения с сильным стоком тепла при малых температурах.

Библиография:: 9 названий.

Исследуя эффект мгновенного сжатия носителя решения задачи Коши для нелинейного вырождающегося параболического уравнения

$$
u_{t}=\operatorname{div}\left(u^{n} \nabla u\right)-u^{\beta}, \quad n \geqslant 0, \quad 0<\beta<1,
$$

с положительной и бесконечно малой при $|x| \rightarrow+\infty$ начальной функцией $\varphi$, доказаны точные локальные оценки для гранищы носителя решений. В случае $n \geqslant 1-\beta$ доказана асимптотически точная формула

$$
\varphi(\xi(t)) \sim((1-\beta) t)^{1 /(1-\beta)}, \quad \varphi(\eta(t)) \sim((1-\beta) t)^{1 /(1-\beta)}, \quad t \rightarrow 0,
$$

где

$$
\xi(t)=\inf \{\rho>0: u(x, t)=0,|x| \geqslant \rho\}, \quad \eta(t)=\sup \{\rho>0: u(x, t)>0,|x|<\rho\} .
$$

1. Введение и основные результаты. Рассмотрим задачу Коши

$$
\begin{gathered}
u_{t}=\operatorname{div}\left(u^{n} \nabla u\right)-u^{\beta}, \quad x \in \mathbb{R}^{N}, \quad t>0, \\
u(x, 0)=u_{0}(x)>0, \quad x \in \mathbb{R}^{N},
\end{gathered}
$$

где $u=u(x, t) \geqslant 0, x \in \mathbb{R}^{N}, t \geqslant 0, n \geqslant 0,0<\beta<1$. Уравнение (2) описывает процессы нелинейной теплопроводности со стоком, одновременно протекающие процессы диффузии и абсорбции, и т.д. [1]. Всюду в дальнейшем будем считать, что вьполнено условие

$$
u_{0}(x)=\varphi(|x|)>0, \quad \varphi \in C^{1}(0,+\infty), \lim _{r \rightarrow+\infty} \varphi(r)=0,
$$

причем никаких ограничений на скорость убывания не накладывается. Решение задачи $(2),(3)$ будем понимать в обобщенном смысле (см. [1]). Очевидно, что при вьполнении условия (4) решение является радиально-симметричным.

Теоремы существования и единственности обобщенных решений, различные теоремы сравнения для задачи (2), (3) установлены в [2]-[4]. Качественному исследованию свойств решений задачи $(2),(3)$ посвящены многие работы. Подробньй обзор имеется в [1], [5]. Интересным является эффект мгновенной локализации (или же эффект мгновенной компактификации носителя решений), обнаруженньй для уравнения (2). 
ОПРЕДЕЛЕНИЕ 1. Пусть выполнено условие (4). Для решения задачи (2), (3) имеет место әффект мәновенной локализации (МЛ), если для любого $t \in(0, \delta], \delta>0$, существует $\eta(t) \in[0,+\infty)$ такое, что $u(x, t)=0$ при $|x| \geqslant \eta(t)$.

Впервые эффект МЛ для уравнения (2) при $N=1, n \geqslant 0,0<\beta<1$ (в случае произвольного $N$ при $n=0,0<\beta<1$ ) был обнаружен авторами [6]. В [7] исследовано влияние коэффициента, зависящего от точек пространства, на эффект МЛ для полулинейного уравнения $u_{t}=u_{x x}-c(x) u^{\beta}$ при степенных начальных функциях. Эффект МЛ в задаче Коши для уравнения $u_{t}=\left(u^{n} u_{x}\right)_{x}+\left(u^{\beta}\right)_{x}$ установлен в [8]. В [9] установлены локальные оценки границы носителя решений задачи $(2),(3)$ при $n=0,0<\beta<1$. Пусть $\xi(t)$ и $\eta(t)$ заданы согласно (1). В [9] доказано, что если $u_{0}(x) \leqslant M(1+|x|)^{-\alpha}, x \in \mathbb{R}^{N}$, $M>0, \alpha>0$, то найдутся числа $T>0$ и $A>0$ такие, что $\xi(t) \leqslant A t^{-1 /(\alpha(1-\beta))}$, $0<t \leqslant T$. Если же $u_{0}(x) \geqslant m(1+|x|)^{-\alpha}, x \in \mathbb{R}^{N}, m>0, \alpha>0$, то найдутся числа $T>0$ и $a>0$ такие, что $\eta(t) \geqslant a t^{-1 /(\alpha(1-\beta))}, 0<t \leqslant T$.

В настоящей работе установлены точные локальные оценки границы носителя решений задачи (2), (3). Доказаны асимптотически точные формулы для границы носителя. В следуюших теоремах сформулированы основные результаты работы.

ТЕОРема 1. Пусть $n \geqslant 1-\beta$, выполнено условие (4), существует $\bar{r}>0$ такое, чmo $\varphi^{\prime}<0$ npu $r \geqslant \bar{r} u$

$$
\sup _{r \geqslant \bar{r}}\left|\left(\varphi^{\gamma}\right)^{\prime \prime}\right|<\infty, \quad \gamma=1-\beta
$$

Тогда для решения задачи (2), (3) имеет место әффект МЛ и для любого достаточно малого $\varepsilon>0$ существует $\delta>0$ такое, что справедливы следующие локальные оченки:

$$
\begin{gathered}
\varphi^{-1}\left(\frac{1}{1-\varepsilon}((1-\beta+\varepsilon) t)^{1 /(1-\beta)}\right) \leqslant \eta(t) \leqslant \xi(t) \leqslant \varphi^{-1}\left(\frac{1}{1+\varepsilon}((1-\beta-\varepsilon) t)^{1 /(1-\beta)}\right) \\
t \in(0, \delta]
\end{gathered}
$$

әде $\varphi^{-1}-$ обратная $к \varphi$ функиия.

СлЕДСТВИЕ 1. При условиях теоремы 1 справедлива следующая асимптотически точная формула:

$$
\xi(t) \sim \varphi^{-1}\left(((1-\beta) t)^{1 /(1-\beta)}\right), \quad \eta(t) \sim \varphi^{-1}\left(((1-\beta) t)^{1 /(1-\beta)}\right), \quad t \rightarrow 0
$$

СлЕДСТВИЕ 2. Пусть выполнены условия теоремы 1. Тогда для любого достаточно малого $\varepsilon>0$ существуют $\rho_{\varepsilon}>0 u \delta>0$ такие, что

$$
\begin{aligned}
& {\left[((1-\varepsilon) \varphi(|x|))^{1-\beta}-(1-\beta+\varepsilon) t\right]_{+}^{1 /(1-\beta)} \leqslant u(x, t)} \\
& \quad \leqslant\left[((1+\varepsilon) \varphi(|x|))^{1-\beta}-(1-\beta-\varepsilon) t\right]_{+}^{1 /(1-\beta)}, \quad(x, t) \in\left\{|x| \geqslant \rho_{\varepsilon}\right\} \times[0, \delta] .
\end{aligned}
$$


ТЕОремА 2. Пусть $0<\beta<1,0 \leqslant n<1-\beta$, функиия $\varphi$ удовлетворяет условиям теоремы 1 с $\gamma=(n+1-\beta) / 2$ и в случае $n=0$ дополнительно выполнено условие

$$
\left(\varphi^{1-\beta}\right)^{\prime \prime}=o(1), \quad r \rightarrow+\infty .
$$

Тогда для решения задачи (2), (3) имеет место эффект МЛ и для любого достаточно малого $\varepsilon>0$ существует $\delta>0$ такое, что справедливы следующие локальные оченки:

$$
\varphi^{-1}\left(\frac{1}{1-\varepsilon}((1-\beta+\varepsilon) t)^{1 /(1-\beta)}\right) \leqslant \eta(t) \leqslant \xi(t) \leqslant \varphi^{-1}\left(k_{1} t^{2 /(n+1-\beta)}\right), \quad t \in(0, \delta]
$$

әде $k_{1}>0$ - произвольная постоянная.

СлЕДСТВИЕ 3. Пусть выполнены условия теоремы 2. Тогда для любого достаточно малого $\varepsilon>0$ существуют $\rho_{\varepsilon}>0 u \delta>0$ такие, что

$$
\begin{aligned}
& {\left[((1-\varepsilon) \varphi(|x|))^{1-\beta}-(1-\beta+\varepsilon) t\right]_{+}^{1 /(1-\beta)} \leqslant u(x, t)} \\
& \quad \leqslant\left[((1+\varepsilon) \varphi(|x|))^{(n+1-\beta) / 2}-k t\right]_{+}^{2 /(n+1-\beta)}, \quad(x, t) \in\left\{|x| \geqslant \rho_{\varepsilon}\right\} \times[0, \delta],
\end{aligned}
$$

əде $k=\left((1+\varepsilon) k_{1}\right)^{(n+1-\beta) / 2}$.

2. Доказательство теоремы 1. Рассмотрим следующую функцию типа бегущей волны:

$$
g(x, t)=f(\xi), \quad \xi=C-t-\int_{0}^{r} v(\eta) d \eta, \quad r=|x|=\left(\sum_{i=1}^{N} x_{i}^{2}\right)^{1 / 2},
$$

где $C>0$ - произвольная постоянная, $v \in C^{1}(0,+\infty),\left|v^{\prime}(r)\right| \leqslant M<+\infty, 0<v(r) \leqslant \mu$, и выполнено условие $\int_{0}^{+\infty} v(r) d r=C$. Возьмем произвольное достаточно малое $\varepsilon>0$ и в качестве $f(\xi)$ рассмотрим функцию

$$
f(\xi)=[(1-\beta-\varepsilon) \xi]_{+}^{1 /(1-\beta)}, \quad \text { где }[\varkappa]_{+}= \begin{cases}\varkappa, & \text { если } \varkappa \geqslant 0, \\ 0, & \text { если } \varkappa<0 .\end{cases}
$$

Функция $g$ удовлетворяет условию

$$
g(x, 0)=\left((1-\beta-\varepsilon)\left(C-\int_{0}^{r} v(\eta) d \eta\right)\right)^{1 /(1-\beta)}, \quad x \in \mathbb{R}^{N} .
$$

Согласно (9) $g(x, 0)>0$ при $x \in \mathbb{R}^{N}$ и $g(x, 0) \rightarrow 0$ при $|x| \rightarrow+\infty$. Однако, в любой момент времени $t \in(0, C]$

$$
g(x, t)=0, \quad|x| \geqslant l(C-t),
$$

где $r=l(z)$ - обратная функция к $z=\int_{0}^{r} v(\eta) d \eta$. Проверим, что $g(x, t)$ является суперрешением уравнения $(2)$ при $(x, t) \in\{|x|>0\} \times\left[0, t_{1}\right]$ для некоторого $t_{1}>0$ :

$$
\begin{aligned}
L g \equiv & g_{t}-\operatorname{div}\left(g^{n} \nabla g\right)+g^{\beta}=-f^{\prime}(\xi)+v^{\prime}(r) f^{n} f^{\prime}+\frac{N-1}{r} v(r) f^{n} f^{\prime}-v^{2}(r)\left(f^{n} f^{\prime}\right)^{\prime}+f^{\beta} \\
\geqslant & -\frac{1-\beta-\varepsilon}{1-\beta}((1-\beta-\varepsilon) \xi)^{\beta /(1-\beta)}-M\left(1-\frac{\varepsilon}{1-\beta}\right)((1-\beta-\varepsilon) \xi)^{(n+\beta) /(1-\beta)} \\
& +\frac{N-1}{r} v(r) \frac{1-\beta-\varepsilon}{1-\beta}((1-\beta-\varepsilon) \xi)^{(n+\beta) /(1-\beta)}+((1-\beta-\varepsilon) \xi)^{\beta /(1-\beta)} \\
& -(1-\beta)^{-2}(1-\beta-\varepsilon)^{2}(n+\beta) \mu^{2}((1-\beta-\varepsilon) \xi)^{(n+2 \beta-1) /(1-\beta)}
\end{aligned}
$$




$$
\begin{aligned}
\geqslant & ((1-\beta-\varepsilon) \xi)^{\beta /(1-\beta)}\left(\varepsilon(1-\beta)^{-1}-M(1-\beta)^{-1}(1-\beta-\varepsilon)((1-\beta-\varepsilon) \xi)^{n /(1-\beta)}\right. \\
& \left.-\mu^{2}(1-\beta)^{-2}(1-\beta-\varepsilon)^{2}(n+\beta)((1-\beta-\varepsilon) \xi)^{(n+\beta-1) /(1-\beta)}\right) .
\end{aligned}
$$

Из (10) следует, что если $n>1-\beta, 0<\beta<1$, то для некоторого $\xi_{0}=\xi_{0}(\varepsilon)>0$

$$
L g \geqslant 0, \quad \xi \in\left[0, \xi_{0}\right]
$$

Если же $n=1-\beta, 0<\beta<1$, то условие (11) также выполняется, если

$$
0<\mu<\varepsilon^{1 / 2}(1-\beta)^{-1 / 2}
$$

Таким образом, если $0<\beta<1, n \geqslant 1-\beta$, то для любого $C \in\left(0, \xi_{0}\right)$ функция $g$ является суперрешением уравнения (2) при $(x, t) \in\{|x|>0\} \times\left[0, t_{1}\right], t_{1} \in(0, C)$. Из условия (4) следует, что для любого $t>0 u(x, t) \rightarrow 0$ при $|x| \rightarrow+\infty$. Обозначим

$$
r_{\varepsilon}(t)=\min \left\{r_{*} \geqslant 0: u(x, t) \leqslant \varepsilon,|x| \geqslant r_{*}\right\} .
$$

Пусть $\rho_{\varepsilon}>0$ удовлетворяет условию $\rho_{\varepsilon} \geqslant \bar{r}$ и

$$
\rho_{\varepsilon} \geqslant \max _{0 \leqslant t \leqslant T} r_{\varepsilon}(t)
$$

для некоторого $T>0$. Обозначим

$$
\begin{gathered}
v_{\varepsilon}(r)=-(1-\beta-\varepsilon)^{-1}\left(((1+\varepsilon) \varphi(r))^{1-\beta}\right)^{\prime}, \quad \rho_{\varepsilon} \leqslant r<+\infty \\
C_{\varepsilon}=(1-\beta-\varepsilon)^{-1}\left((1+\varepsilon) \varphi\left(\rho_{\varepsilon}\right)\right)^{1-\beta} .
\end{gathered}
$$

По выбранному $\varepsilon>0$ сделаем $\rho_{\varepsilon}$ настолько большим, чтобы $C_{\varepsilon} \in\left(0, \xi_{0}\right]$. Нетрудно проверить, что

$$
\int_{\rho_{\varepsilon}}^{+\infty} v_{\varepsilon}(\eta) d \eta=C_{\varepsilon}
$$

Так как по условию теоремы $v_{\varepsilon}^{\prime}(r)$ является ограниченной функцией при $r \in\left[\rho_{\varepsilon},+\infty\right)$, из (13) следует

$$
\lim _{r \rightarrow+\infty} v_{\varepsilon}(r)=0
$$

Ввиду (14) для некоторого $r_{1} 0<v_{\varepsilon}(r) \leqslant \mu$ при $r \geqslant r_{1}$, где $\mu>0$ удовлетворяет условию (12). Будем считать, что $\rho_{\varepsilon} \geqslant r_{1}$.

Теперь рассмотрим функцию

$$
g_{\varepsilon}(x, t)=\left((1-\beta-\varepsilon)\left[C_{\varepsilon}-t-\int_{\rho_{\varepsilon}}^{|x|} v_{\varepsilon}(\eta) d \eta\right]_{+}\right)^{1 /(1-\beta)}
$$


Как это сделано выше, можно доказать, что существует $t_{1} \in(0, T)$ такое, что $g_{\varepsilon}$ является суперрешением уравнения (2) при $(x, t) \in B_{\rho_{\varepsilon}} \times\left[0, t_{1}\right]$, где $B_{\rho_{\varepsilon}}=\left\{x \in \mathbb{R}^{N}:|x| \geqslant \rho_{\varepsilon}\right\}$. Из (13) следует, что $g_{\varepsilon}(x, 0)>0$ при $|x| \geqslant \rho_{\varepsilon}$. Далее имеем

$$
\begin{aligned}
g_{\varepsilon}(x, 0)= & \left((1-\beta-\varepsilon)\left(C_{\varepsilon}-\int_{\rho_{\varepsilon}}^{|x|} v_{\varepsilon}(\eta) d \eta\right)\right)^{1 /(1-\beta)} \\
= & \left((1-\beta-\varepsilon) \int_{|x|}^{+\infty} v_{\varepsilon}(\eta) d \eta\right)^{1 /(1-\beta)}=(1+\varepsilon) \varphi(|x|)>\varphi(|x|), \quad|x| \geqslant \rho_{\varepsilon}, \\
& \left.g_{\varepsilon}(x, t)\right|_{|x|=\rho_{\varepsilon}}=\left((1-\beta-\varepsilon)\left[C_{\varepsilon}-t\right]_{+}\right)^{1 /(1-\beta)}, \quad 0 \leqslant t \leqslant t_{1} .
\end{aligned}
$$

Так как

$$
\left.g_{\varepsilon}(x, 0)\right|_{|x|=\rho_{\varepsilon}}=\left((1-\beta-\varepsilon) C_{\varepsilon}\right)^{1 /(1-\beta)}=(1+\varepsilon) \varphi\left(\rho_{\varepsilon}\right)>\varphi\left(\rho_{\varepsilon}\right),
$$

существует $\delta_{1}>0$ такое, что

$$
\left.g_{\varepsilon}(x, t)\right|_{|x|=\rho_{\varepsilon}}=\left.u(x, t)\right|_{|x|=\rho_{\varepsilon}} \quad t \in\left[0, \delta_{1}\right] .
$$

Обозначим $\delta_{2}=\min \left\{\delta_{1}, t_{1}\right\}$. Учитьвая $(15),(16)$ и применяя теорему сравнения для уравнения $(2)$, получим оценку $0 \leqslant u(x, t) \leqslant g_{\varepsilon}(x, t),(x, t) \in B_{\rho_{\varepsilon}} \times\left[0, \delta_{2}\right]$. Отсюда и из свойств $g_{\varepsilon}$ следует, что

$$
u(x, t)=0, \quad|x| \geqslant l_{\varepsilon}\left(C_{\varepsilon}-t\right), \quad 0<t \leqslant \delta_{2},
$$

где $r=l_{\varepsilon}(z)$ - обратная функция к функции $z=\int_{\rho_{\varepsilon}}^{r} v_{\varepsilon}(\eta) d \eta$. Так как

$$
\int_{\rho_{\varepsilon}}^{r} v_{\varepsilon}(\eta) d \eta=C_{\varepsilon}-(1-\beta-\varepsilon)^{-1}((1+\varepsilon) \varphi(r))^{1-\beta}
$$

имеем

$$
l_{\varepsilon}\left(C_{\varepsilon}-t\right)=\varphi^{-1}\left((1+\varepsilon)^{-1}((1-\beta-\varepsilon) t)^{1 /(1-\beta)}\right) .
$$

Поэтому из (17) следует, что

$$
u(x, t)=0, \quad|x| \geqslant \varphi^{-1}\left((1+\varepsilon)^{-1}((1-\beta-\varepsilon) t)^{1 /(1-\beta)}\right), \quad t \in\left(0, \delta_{2}\right),
$$

откуда следует наличие эффекта МЛ и правая часть оценки (5).

Перейдем к доказательству левой части оценки (5). Схема доказательства такая же. Рассмотрим функцию $g$ из (8), где $C, v$ такие же, как и в (8), (9). В качестве $f$ возьмем функцию

$$
f(\xi)=[(1-\beta+\varepsilon) \xi]_{+}^{1 /(1-\beta)} .
$$

Проверим, что $g$ является субрешением уравнения $(2)$ при $(x, t) \in B_{r_{0}} \times\left[0, t_{1}\right]$ для некоторых $t_{1}>0$ и $r_{0}>0$ :

$$
\begin{aligned}
L g= & -f^{\prime}(\xi)+v^{\prime}(r) f^{n} f^{\prime}+r^{-1}(N-1) v(r) f^{n} f^{\prime}-v^{2}(r)\left(f^{n} f^{\prime}\right)^{\prime}+f^{\beta} \\
\leqslant & -f^{\prime}+M f^{n} f^{\prime}+r_{0}^{-1}(N-1) \mu f^{n} f^{\prime}+f^{\beta} \\
= & -\left(1+\frac{\varepsilon}{1-\beta}\right)((1-\beta+\varepsilon) \xi)^{\beta /(1-\beta)}+M_{1}\left(1+\frac{\varepsilon}{1-\beta}\right)((1-\beta+\varepsilon) \xi)^{(n+\beta) /(1-\beta)} \\
& +((1-\beta+\varepsilon) \xi)^{\beta /(1-\beta)} \\
= & ((1-\beta+\varepsilon) \xi)^{\beta /(1-\beta)}\left\{-\frac{\varepsilon}{1-\beta}+M_{1}\left(1+\frac{\varepsilon}{1-\beta}\right)((1-\beta+\varepsilon) \xi)^{n /(1-\beta)}\right\},
\end{aligned}
$$


где $M_{1}=M+(\mu(N-1)) / r_{0}$. Из (18) следует, что существует $\xi_{1}=\xi_{1}(\varepsilon)>0$ такое, что

$$
L g \leqslant 0, \quad \xi \in\left[0, \xi_{1}\right] .
$$

Таким образом, для любого $C \in\left(0, \xi_{1}\right)$ функция $g$ является субрешением уравнения $(2)$ при $(x, t) \in B_{r_{0}} \times\left[0, t_{1}\right], t_{1} \in(0, C)$.

Как и вьше, определим точку $\rho_{\varepsilon} \geqslant \max \left\{r_{0}, \bar{r}\right\}$. Пусть

$$
\begin{gathered}
v_{\varepsilon}(r)=-(1-\beta+\varepsilon)^{-1}\left(((1-\varepsilon) \varphi(r))^{1-\beta}\right)^{\prime}, \quad \rho_{\varepsilon} \leqslant r<+\infty \\
C_{\varepsilon}=(1-\beta+\varepsilon)^{-1}\left((1-\varepsilon) \varphi\left(\rho_{\varepsilon}\right)\right)^{1-\beta} .
\end{gathered}
$$

Точно также доказьваются условия (13), (14). Пусть

$$
g_{\varepsilon}(x, t)=\left((1-\beta+\varepsilon)\left[C_{\varepsilon}-t-\int_{\rho_{\varepsilon}}^{|x|} v_{\varepsilon}(\eta) d \eta\right]_{+}\right)^{1 /(1-\beta)} .
$$

Аналогично тому, как это сделано вьше, можно доказать, что $g$ является субрешением уравнения $(2)$ при $(x, t) \in B_{\rho_{\varepsilon}} \times\left[0, t_{1}\right], t_{1}>0$. Из (13) следует, что $g_{\varepsilon}(x, 0)>0$ при $|x| \geqslant \rho_{\varepsilon}$. Далее имеем

$$
\begin{gathered}
g_{\varepsilon}(x, 0)=(1-\varepsilon) \varphi(|x|)<\varphi(|x|), \quad|x| \geqslant \rho_{\varepsilon}, \\
\left.g_{\varepsilon}(x, t)\right|_{|x|=\rho_{\varepsilon}}=\left((1-\beta+\varepsilon)\left[C_{\varepsilon}-t\right]_{+}\right)^{1 /(1-\beta)}, \quad 0 \leqslant t \leqslant t_{1} .
\end{gathered}
$$

Tak как

$$
\left.g_{\varepsilon}(x, 0)\right|_{|x|=\rho_{\varepsilon}}=\left((1-\beta+\varepsilon) C_{\varepsilon}\right)^{1 /(1-\beta)}=(1-\varepsilon) \varphi\left(\rho_{\varepsilon}\right)<\varphi\left(\rho_{\varepsilon}\right),
$$

существует $\delta_{1}>0$ такое, что

$$
\left.g_{\varepsilon}(x, t)\right|_{|x|=\rho_{\varepsilon}} \leqslant\left. u(x, t)\right|_{|x|=\rho_{\varepsilon}}, \quad t \in\left[0, \delta_{1}\right] .
$$

Обозначим $\delta_{2}=\min \left\{\delta_{1}, t_{1}\right\}$. Учитьвая $(19),(20)$ и применяя теорему сравнения для уравнения (2), получим оценку

$$
u(x, t) \geqslant g_{\varepsilon}(x, t), \quad(x, t) \in B_{\rho_{\varepsilon}} \times\left[0, \delta_{2}\right] .
$$

Отсюда и из свойств $g_{\varepsilon}$ следует, что

$$
u(x, t)>0, \quad \rho_{\varepsilon} \leqslant|x|<l_{\varepsilon}\left(C_{\varepsilon}-t\right), \quad 0 \leqslant t \leqslant \delta_{2},
$$

где $r=l_{\varepsilon}(z)$ - обратная функция к $z=\int_{\rho_{\varepsilon}}^{r} v_{\varepsilon}(\eta) d \eta$. Отметим, что если $u(x, 0)>0$ при $|x| \leqslant \rho_{\varepsilon}$, то, уменьшая в случае необходимости $\delta_{2}$, всегда можно добиться выполнения условия

$$
u(x, t)>0, \quad|x|<l_{\varepsilon}\left(C_{\varepsilon}-t\right), \quad 0 \leqslant t \leqslant \delta_{2} .
$$

Очевидно,

$$
l_{\varepsilon}\left(C_{\varepsilon}-t\right)=\varphi^{-1}\left(\frac{1}{1-\varepsilon}((1-\beta+\varepsilon) t)^{1 /(1-\beta)}\right) .
$$


Поэтому из (21) следует, что

$$
u(x, t)>0, \quad|x|<\varphi^{-1}\left(\frac{1}{1-\varepsilon}((1-\beta+\varepsilon) t)^{1 /(1-\beta)}\right), \quad t \in\left(0, \delta_{2}\right] .
$$

Таким образом, оценка (5) полностью доказана. Теорема доказана.

Так как $\varphi$ является строго монотонно убывающей при $|x| \rightarrow+\infty$, из (5) следует

$$
\frac{1}{1+\varepsilon}((1-\beta-\varepsilon) t)^{1 /(1-\beta)} \leqslant \varphi(\xi(t)) \leqslant \frac{1}{1-\varepsilon}((1-\beta+\varepsilon) t)^{1 /(1-\beta)}, \quad t \in(0, \delta]
$$

или

$$
\begin{aligned}
\frac{1}{1+\varepsilon} \frac{((1-\beta-\varepsilon) t)^{1 /(1-\beta)}}{((1-\beta) t)^{1 /(1-\beta)}} & \leqslant \frac{\varphi(\xi(t))}{((1-\beta) t)^{1 /(1-\beta)}} \\
& \leqslant \frac{1}{1-\varepsilon} \frac{((1-\beta+\varepsilon) t)^{1 /(1-\beta)}}{((1-\beta) t)^{1 /(1-\beta)}}, \quad t \in(0, \delta] .
\end{aligned}
$$

Переходя к пределу при $t \rightarrow 0$, получаем

$$
\frac{1}{1+\varepsilon}\left(\frac{1-\beta-\varepsilon}{1-\beta}\right)^{1 /(1-\beta)} \leqslant \lim _{t \rightarrow 0} \frac{\varphi(\xi(t))}{((1-\beta) t)^{1 /(1-\beta)}} \leqslant \frac{1}{1-\varepsilon}\left(\frac{1-\beta+\varepsilon}{1-\beta}\right)^{1 /(1-\beta)} .
$$

Ввиду произвольности $\varepsilon>0$

$$
\lim _{t \rightarrow 0} \frac{\varphi(\xi(t))}{((1-\beta) t)^{1 /(1-\beta)}}=1
$$

или

$$
\varphi(\xi(t)) \sim((1-\beta) t)^{1 /(1-\beta)}, \quad t \rightarrow 0,
$$

откуда следует асимптотическая формула (6) для $\xi(t)$. Аналогично доказьвается асимптотическая формула для $\eta(t)$. Следствие доказано. Следствие 2 непосредственно следует из доказательства теоремы 1.

3. Доказательство теоремы 2. Отметим, что левая часть оценки (7) доказана нами в теореме 1. Действительно, как нетрудно заметить, при доказательстве левой части оценки (5) мы использовали только лишь условие $n>0$ (см. (18)). Остается рассмотреть случай $n=0$. В этом случае второе слагаемое в фигурных скобках из (18) не равно 0 при $\xi=0$. Однако, опираясь на условие теоремы 2 , по любому заданному достаточно малому $\varepsilon>0$ точка $\rho_{\varepsilon}$ может быть выбрана так, что

$$
M_{1}=M+(N-1) \rho_{\varepsilon}^{-1} \mu \equiv \sup _{r \geqslant \rho_{\varepsilon}}\left(\left|v_{\varepsilon}^{\prime}(r)\right|+(N-1) \rho_{\varepsilon}^{-1}\left|v_{\varepsilon}(r)\right|\right)<\varepsilon(1-\beta+\varepsilon)^{-1}
$$

В этом случае из $(18)$ опять следует, что $L g \leqslant 0$ при $\xi \in\left[0, \xi_{1}\right]$ для некоторого $\xi_{1}>0$. Следовательно, нам достаточно доказать правую часть оценки (7). Схема доказательства такая же, как и в доказательстве теоремы 1. 
Рассмотрим функцию $g$ из (8), где $C, v$ такие же, как и в $(8),(9)$. В качестве $f$ возьмем функцию

$$
f(\xi)=(k \xi)^{2 /(n+1-\beta)}, \quad k>0 .
$$

Проверим, что $g$ является суперрешением уравнения $(2)$ при $(x, t) \in\{|x|>0\} \times\left[0, t_{1}\right]$ для некоторого $t_{1}>0$ :

$$
\begin{aligned}
L g= & -f^{\prime}(\xi)+v^{\prime}(r) f^{n} f^{\prime}+(N-1) r^{-1} v(r) f^{n} f^{\prime}-v^{2}(r)\left(f^{n} f^{\prime}\right)^{\prime}+f^{\beta} \\
\geqslant & -2 k(n+1-\beta)^{-1}(k \xi)^{(1+\beta-n) /(n+1-\beta)}-2 k M(n+1-\beta)^{-1}(k \xi)^{(n+1+\beta) /(n+1-\beta)} \\
& -2 k^{2} \mu^{2}(n+1+\beta)(n+1-\beta)^{-2}(k \xi)^{2 \beta /(n+1-\beta)}+(k \xi)^{2 \beta /(n+1-\beta)} \\
= & (k \xi)^{2 \beta /(n+1-\beta)}\left(1-\frac{2 k}{n+1-\beta}(k \xi)^{(1-\beta-n) /(n+1-\beta)}\right. \\
& \left.-\frac{2 k^{2} M}{n+1-\beta} \xi-\frac{2 k^{2} \mu^{2}(n+1+\beta)}{(n+1-\beta)^{2}}\right) .
\end{aligned}
$$

Пусть выполнено условие

$$
0<\mu^{2}<\left(2 k^{2}(n+1+\beta)\right)^{-1}(n+1-\beta)^{2} .
$$

В этом случае из $(22)$ следует, что для некоторого $\xi_{0}=\xi_{0}(\varepsilon)>0$

$$
L g \geqslant 0, \quad \xi \in\left[0, \xi_{0}\right]
$$

Таким образом, для любого $C \in\left(0, \xi_{0}\right)$ функция $g$ является суперрешением уравнения (2) при $\{|x|>0\} \times\left[0, t_{1}\right], t_{1} \in(0, C)$.

Аналогично тому, как это сделано в доказательстве теоремы 1 , определим $\rho_{\varepsilon}>0$. Пусть

$$
\begin{gathered}
v_{\varepsilon}(r)=-k^{-1}\left(((1+\varepsilon) \varphi(r))^{(n+1-\beta) / 2}\right)^{\prime}, \quad \rho_{\varepsilon} \leqslant r<+\infty \\
C_{\varepsilon}=k^{-1}\left((1+\varepsilon) \varphi\left(\rho_{\varepsilon}\right)\right)^{(n+1-\beta) / 2}
\end{gathered}
$$

Сделаем $\rho_{\varepsilon}$ настолько большим, чтобы $C_{\varepsilon} \in\left[0, \xi_{0}\right]$. Нетрудно проверить, что для выбранньг $v_{\varepsilon}$ и $C_{\varepsilon}$ вьполняются условия (13) и (14).

Ввиду (14) для некоторого $r_{1} 0 \leqslant v_{\varepsilon} \leqslant \mu$ при $r \geqslant r_{1}$, где $\mu>0$ удовлетворяет условию (23). Будем считать, что $\rho_{\varepsilon} \geqslant r_{1}$. Далее рассмотрим функцию

$$
g_{\varepsilon}(x, t)=\left(k\left[C_{\varepsilon}-t-\int_{\rho_{\varepsilon}}^{|x|} v_{\varepsilon}(\eta) d \eta\right]_{+}\right)^{2 /(n+1-\beta)} .
$$

Аналогично тому, как это сделано вьше, можно доказать, что существует $t_{1}>0$ такое, что $g_{\varepsilon}(x, t)$ является суперрешением уравнения $(2)$ при $(x, t) \in B_{\rho_{\varepsilon}} \times\left[0, t_{1}\right]$. Далееимеем

$$
\begin{gathered}
g_{\varepsilon}(x, 0)=(1+\varepsilon) \varphi(|x|)>\varphi(|x|), \quad|x| \geqslant \rho_{\varepsilon}, \\
\left.g_{\varepsilon}(x, t)\right|_{|x|=\rho_{\varepsilon}}=\left(k\left[C_{\varepsilon}-t\right]_{+}\right)^{2 /(n+1-\beta)}, \quad 0 \leqslant t \leqslant t_{1} .
\end{gathered}
$$


Так как

$$
\left.g_{\varepsilon}(x, 0)\right|_{|x|=\rho_{\varepsilon}}=\left(k C_{\varepsilon}\right)^{2 /(n+1-\beta)}=(1+\varepsilon) \varphi\left(\rho_{\varepsilon}\right)>\varphi\left(\rho_{\varepsilon}\right),
$$

существует $\delta_{1}>0$ такое, что

$$
\left.g_{\varepsilon}(x, t)\right|_{|x|=\rho_{\varepsilon}} \geqslant\left. u(x, t)\right|_{|x|=\rho_{\varepsilon}}, \quad t \in\left[0, \delta_{1}\right] .
$$

Обозначим $\delta_{2}=\min \left\{\delta_{1}, t_{1}\right\}$. Учитывая $(24),(25)$ и теорему сравнения для уравнения (2), получим оценку

$$
0 \leqslant u(x, t) \leqslant g_{\varepsilon}(x, t), \quad(x, t) \in B_{\rho_{\varepsilon}} \times\left[0, \delta_{2}\right] .
$$

Отсюда и из свойств $g_{\varepsilon}$ следует, что

$$
u(x, t)=0, \quad|x| \geqslant l_{\varepsilon}\left(C_{\varepsilon}-t\right), \quad 0<t \leqslant \delta_{2},
$$

где $r=l_{\varepsilon}(z)$ - обратная функция к

$$
z=\int_{\rho_{\varepsilon}}^{r} v_{\varepsilon}(\eta) d \eta=C_{\varepsilon}-k^{-1}((1+\varepsilon) \varphi(r))^{(n+1-\beta) / 2},
$$

т.e.

$$
l_{\varepsilon}\left(C_{\varepsilon}-t\right)=\varphi^{-1}\left(\frac{1}{1+\varepsilon}(k t)^{2 /(n+1-\beta)}\right) .
$$

Таким образом, из (26) получаем, что

$$
u(x, t)=0, \quad|x| \geqslant \varphi^{-1}\left(k_{1} t^{2 /(n+1-\beta)}\right), \quad t \in(0, \delta],
$$

откуда следует наличие эффекта МЛ и правая часть оценки (7).

Очевидно, что длина временного интервала $\delta$ зависит от $k_{1}$. Теорема доказана.

Следствие 3 непосредственно вытекает из доказательства теоремы 2 .

\section{СПИСОК ЦИТИРОВАННОЙ ЛИТЕРАТУРЫ}

[1] Калашников А. С. Некоторые вопросы качественной теории нелинейных вырождающихся параболических уравнений второго порядка // УМН. 1987. Т. 42. № 2(254). С. 135-176.

[2] Кершнер Р. О некоторых свойствах обобщенных решений квазилинейных вырождающихся параболических уравнений // Acta Math. Hungar. 1978. V. 32. P. 301-330.

[3] Sacks P.E. The initial and boundary value problem for a class of degenerate parabolic equations // Comm. Partial Differential Equations. 1983. V. 8. P. 693-733.

[4] Bertch M.A. A class of degenerate diffusion equations with a singular term // Nonlinear Anal. 1983. V. 7. P. 117-127.

[5] Самарский А. А., Галактионов В.А., Курдюмов С. П., Михайлов А. П. Режимы с обострением в задачах для квазилинейных параболических уравнений. М.: Наука, 1987.

[6] Evans L.C., Knerr B. K. Instantaneous shrinking of the support of nonnegative solutions to certain nonlinear parabolic equations and variational inequalities // Illinois J. Math. 1979. V. 23. № 1. P. 153-166.

[7] Калашников А. С. О зависимости свойств решений параболических уравнений в неограниченных областях от поведения коэффициентов на бесконечности // Матем. сб. 1984. Т. 125. №3. C. 398-409.

[8] Gilding B.H., Kersner R. Instantaneous shrinking in nonlinear diffusion convection // Proc. Amer. Math. Soc. 1990. V. 109. P. 385-394.

[9] Калашников А. С. О поведении вблизи начальной гиперплоскости решений задачи Коши для параболических систем с нелинейной диссипацией // Тр. семин. им. И. Г. Петровского. 1992. T. 16. C. $106-113$.

Бакинский государственный университет им. М.Э. Расулзаде 